\title{
CONDIÇÕES HIGIÊNICAS SANITÁRIAS DA PRODUÇÃO DO CALDO DE CANA EM CURITIBA, PARANÁ
}

(Sanitary hygienic conditions of sugarcane juice production in Curitiba, Parana)

Lew Kan Sprenger, Larissa Wünsche Risolia

\begin{abstract}
${ }^{1}$ Correspondência: lew.sprenger@gmail.com
RESUMO: O caldo de cana, bebida vegetal apreciada pela população brasileira, comercializado por ambulantes pode oferecer riscos à saúde dos consumidores, se produzido de maneira inadequada. Este estudo teve por objetivo verificar se os vendedores de caldo de cana estão seguindo à Resolução RDC no 218, de 29 de julho de 2005 da ANVISA, que normatiza os procedimentos higiênicos sanitários para a produção deste produto. Foram entrevistados 47 vendedores, distribuídos em 17 bairros da cidade de Curitiba, durante os meses de outubro e novembro de 2014. Foi utilizado um questionário fechado, montado baseado na referida Resolução, onde as respostas possíveis eram sim ou não. Este foi previamente testado e validado por meio de um estudo piloto, sendo a aplicação, realizada por uma única pessoa treinada para coletar as informações e capaz de suprir supostas dúvidas, contudo, sem influenciar nas respostas dos entrevistados. Nenhum garapeiro entrevistado, $0 \%$ (0/47), seguiu todos os itens da lei, sendo a falta de asseio pessoal observada em todos os casos 100\% (47/47). Conclui-se que os produtores de caldo de cana de Curitiba não estão obedecendo a resolução vigente, seja por desconhecimento do regulamento ou estarem agindo de má fé. Esta atitude expõem os consumidores à possíveis doenças transmissíveis por alimentos.
\end{abstract}

Palavras-chave: DTAs; garapa; microbiologia de alimentos

ABSTRACT: The sugar cane juice, vegetable drink enjoyed by brazilian population, sold by street can pose risks to consumer health if improperly produced. This study aimed to verify if the sugarcane juice vendors are following the Resolution RDC No. 218 of July 29 2005, ANVISA, which regulates the hygienic-sanitary procedures for the production of this drink. Were interviewed 47 points, distributed in 17 neighborhoods in the city of Curitiba, during the months of October and November 2014. A closed questionnaire was used, based on the Resolution, where possible answers were yes or no. This was previously tested and validated through a pilot study, and the application made by one trained person to collect information and able to meeting doubts, however, without influencing the respondents' answers. None interviewed, 0\% (0/47), respected all the items of the law, and the lack of personal hygiene observed in all cases $100 \%$ (47/47). In conclusion, Curitiba sugarcane juice producers are not obeying the current resolution, either through ignorance of the Regulation or by are acting in bad faith. This attitude expose the consumers to possibles diseases transmitted by food.

Key Words: food microbiology; foodborne diseases; garapa 


\section{INTRODUÇÃO}

Atualmente, devido a mudanças impostas pelo estilo de vida, muitas pessoas estão se alimentando fora dos domicílios. Nos grandes centros urbanos, os habitantes gastam em média $25,74 \%$ da renda familiar com alimentação em estabelecimentos fixos ou no comercio alimentício ambulante (Leal, 2010). Apesar da praticidade e agilidade encontrados, muitas vezes a qualidade higiênico-sanitário é precária (Genta et al., 2008). No Brasil, pastel, sanduíche, pamonha, churros e caldo de cana são alimentos comercializados por ambulantes que apresentam potencial moderado a alto de contaminação por micro-organismos (Oliveira et al., 2006).

O caldo de cana, também conhecido como garapa, bebida produzido a partir da cana de açúcar, é considerado popularmente um produto altamente nutritivo, de sabor agradável e de baixo custo, fatores que a fazem ser muito consumido (Gandra et al., 2007). A cana pode possuir sujidades e microrganismos em seus colmos e folhas, principalmente (Norberg et al., 2012). Contudo, durante a extração há grande chance de contaminação, devido à falta de higiene dos equipamentos, contaminação ambiental ou humana e condições inadequadas de manipulação (Panza et al., 2006). Além disso, a garapa possui diversos nutrientes e condições propícias para o desenvolvimento de micro-organismos patogênicos (França, 2005).

Visando diminuir os problemas de contaminação e possíveis veiculações de doenças transmissíveis por alimentos (DTAs), a Agência Nacional de Vigilância Sanitária (ANVISA) criou a Resolução RDC $n^{\circ} 218$, de 29 de julho de 2005. Nesta está disposta o Regulamento Técnico de Procedimentos Higiênico-Sanitários para Manipulação de Alimentos e Bebidas Preparadas com Vegetais, estando também incluído o caldo de cana (BRASIL, 2005). Entretanto, devido à falta de fiscalização por parte dos órgãos sanitários responsáveis, muitas vezes os comerciantes não obedecem as leis, seja por falta de conhecimento ou por agirem de má fé (Santos et al., 2010). Além disso, também há falta de informação e treinamento acerca dos cuidados higiênico sanitários que devem ser tomados durante todo o processo, desde a manipulação da matéria prima até a entrega do produto final. Estes procedimentos, se forem corretamente implantados na cadeia produtiva, diminuem em mais de $50 \%$ os riscos do contágio com DTAs (Pereira et al., 2009).

O objetivo do presente trabalho foi verificar se os itens constantes na RDC $n^{\circ}$ 218, de 29 de julho de 2005 estão sendo aplicados pelos garapeiros.

\section{MATERIAL E MÉTODOS}

Durante os meses de outubro e novembro de 2014, foram vistoriados 47 estabelecimentos vendedores de caldo de cana, localizados em 21 bairros da cidade de Curitiba, Paraná. A coleta de dados foi realizada por meio da aplicação de um questionário fechado composto com leis aplicáveis da Resolução RDC no 218, de 29 de julho de 2005 (BRASIL, 2005) aos produtores de garapa (Tabela 1). Este questionário, foi previamente testado $\mathrm{e}$ validado por meio de um estudo piloto, sendo a aplicação, realizada por uma única pessoa treinada para coletar as informações e capaz de suprir supostas dúvidas, contudo, sem influenciar nas respostas dos entrevistados.

Todos os participantes foram previamente avisado sobre os propósitos e objetivos do estudo, sendo que os mesmo, quando em livre e espontânea vontade, responderam participaram da pesquisa.

Os dados foram processados no software Statistical Package for Social Sciences - SPSS 16.0, sendo elaboradas tabelas para melhor visualização e apresentação dos mesmos.

\section{RESULTADOS E DISCUSSÃO}

Quanto a análise dos itens sobre aquisição, recebimento e 
armazenamento das matérias-primas, ingredientes, embalagens e insumos que constavam na RDC $\mathrm{n}^{\circ} 218$, de 29 de julho de 2005 (BRASIL, 2005), os resultados encontram-se na Tabela 1. Uma falha observada foi quanto a falta de controle de temperatura no transporte da cana de açúcar, quando apenas 4,3\% (2/47) dos produtores disseram que este item era obedecido. Esta erro também ocorreu no acondicionamento das matérias-primas, quando estas estavam sob a guarda dos garapeiros, 19,1\% (9/47). $\mathrm{O}$ controle da temperatura durante $\mathrm{o}$ armazenamento, é fundamental para reduzir a respiração, a produção de etileno e a transpiração, processos que causam deteriorações fisiológicas, bem como colabora para a diminuição da microbiota. Colmos estocados a temperatura ambiente ou refrigerados possuem alta proliferação de bolores e leveduras, podendo gerar produtos com alterações sensoriais perceptíveis (Alves et al., 2008). Não existe valor máximo de bolores e leveduras fixados na RDC n 12 , de 02 de janeiro de 2001 (BRASIL, 2001), resolução vigente sobre o assunto, entretanto contagens acima de $10^{3}$ UFC $\mathrm{mL}^{-1}$ produzem alterações sensoriais (Jay, 1992). O único método de estocar a matéria-prima, sem que ocorra alterações físico-químicas e proliferação de microrganismos é o congelamento (Alves et al., 2008).

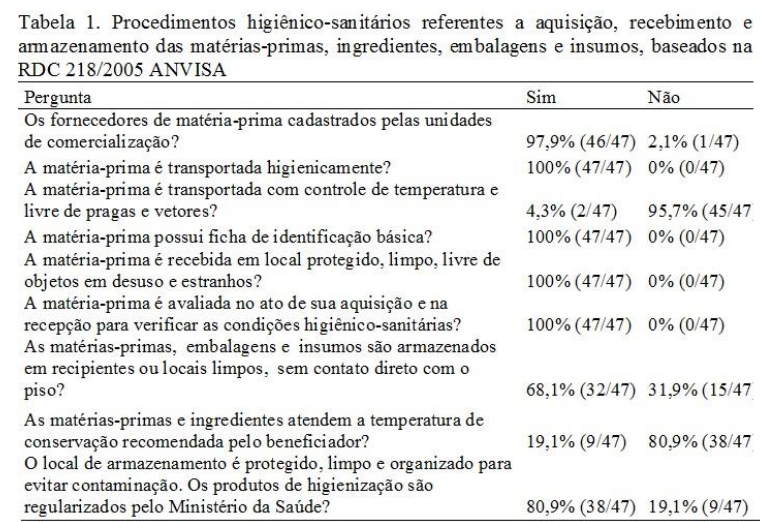

\section{No}

armazenamento

dos ingredientes, $68,1 \%(32 / 47)$ os abrigavam em condições higiénicas e sob proteção de perigos biológicos, físicos e químicos. Este é um problema que ocorre em diversas localidades e atinge todos as categorias de alimentos comercializados. A contaminação pode atingir a matéria-prima, que ao ser processado irá causar DTAs em diversos consumidores (Odorizzi et al., 2011). Os vendedores devem seguir rigorosos processos higiênicos visando evitar problemas alimentares em seus clientes, todavia isto é pouco observado (Almeida e Pena, 2014).

$\mathrm{Na}$ higiene dos manipuladores (Tabela 2), observando o conjunto de hábitos, nenhum $0 \%(0 / 47)$ trabalhador demonstrou possuir completo asseio, segundo as normas estabelecidas. O manuseio dos garapeiros podem interferir diretamente na qualidade e segurança do alimento, nas diferentes fases de elaboração, mesmo se as outras etapas sejam realizadas higienicamente (Andreotti et al., 2007). Cerca de 60\% das enfermidades de origem alimentar causadas por microrganismos patogênicos, sendo que os manipuladores são responsáveis em $26 \%$ dos surtos (Silva, 2013). A contaminação ocorre principalmente nas etapas de manipulação e preparo dos alimentos (Souza, 2006).

Tabela 2. Procedimentos higiênico-sanitários referentes aos manipuladores de alimentos, baseados na RDC 218/2005 ANVISA

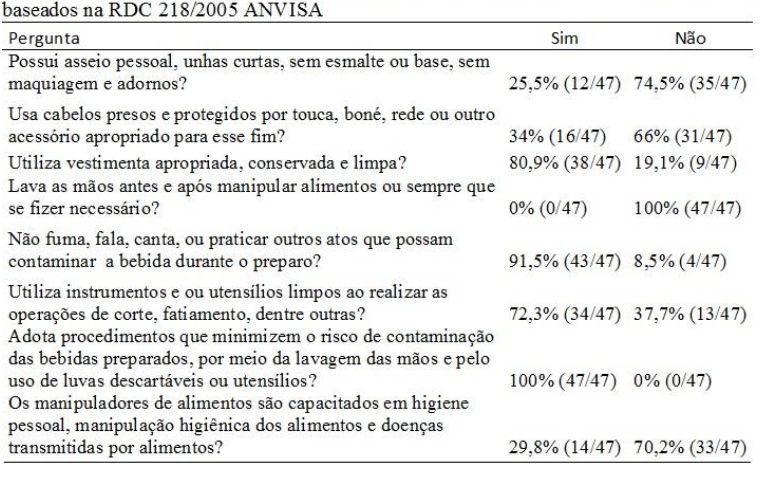

Somente $29,8 \% \quad(14 / 47) \quad$ dos comerciantes afirmaram ter realizado algum tipo de treinamento sobre higiene pessoal, manipulação higiênica dos alimentos e DTAs. Isso demonstra uma falha do sistema de vigilância sanitária municipal, que deveria promover cursos para fornecer subsídios técnicos e científicos para que os garapeiros utilizem no dia-a-dia. Também deve haver a inspeção dos produtores, 
visando identificar quais reciclam frequentemente seus conhecimentos (Torres et al., 2006). Aliando a oferta regular de oficinas de educação em saúde com a fiscalização da aplicação destas noções, diversos casos de contaminação por alimentos podem ser evitados (Alves et al., 2008). Logo, os treinamentos são uma excelente ferramenta para a melhoria da qualidade higiênico-sanitária dos produtos vegetais (Panza et al., 2006; Andreotti et al., 2007).

As respostas dos itens relativos ao preparo e exposição a venda de alimentos e bebidas estão dispostos na Tabela 3. Nenhum local $0 \%(0 / 47)$ demonstrou possuir proteção contra o contato de pragas e vetores. Apenas em 23,4\% (11/47) dos locais visitados, foram visualizados lixeiras com tampas para armazenar os resíduos, visando evitar a presença de vetores e pragas. Esta questão é muito importante, pois existem doenças de extrema relevância que podem ser transmitidas aos consumidores se insetos forem moídos junto a cana de açúcar (Ianni e Mady, 2005). Em surtos de doença de Chagas ocorridos na Paraíba e noutro em Santa Catarina, o veículo carreador do parasita foi o caldo de cana, levado à distância e tomado fora do local de origem da contaminação Sendo que no segundo caso ocorreram a morte ao menos cinco pessoas (Steidel et al., 2005; Cardoso et al., 2006). Esta situação demonstra a importância de prevenir a presença dos vetores na área de produção, tendo em vista que as referidas situações alertam para ocorrência de problemas com grandes dimensões (Duarte et al., 2011).

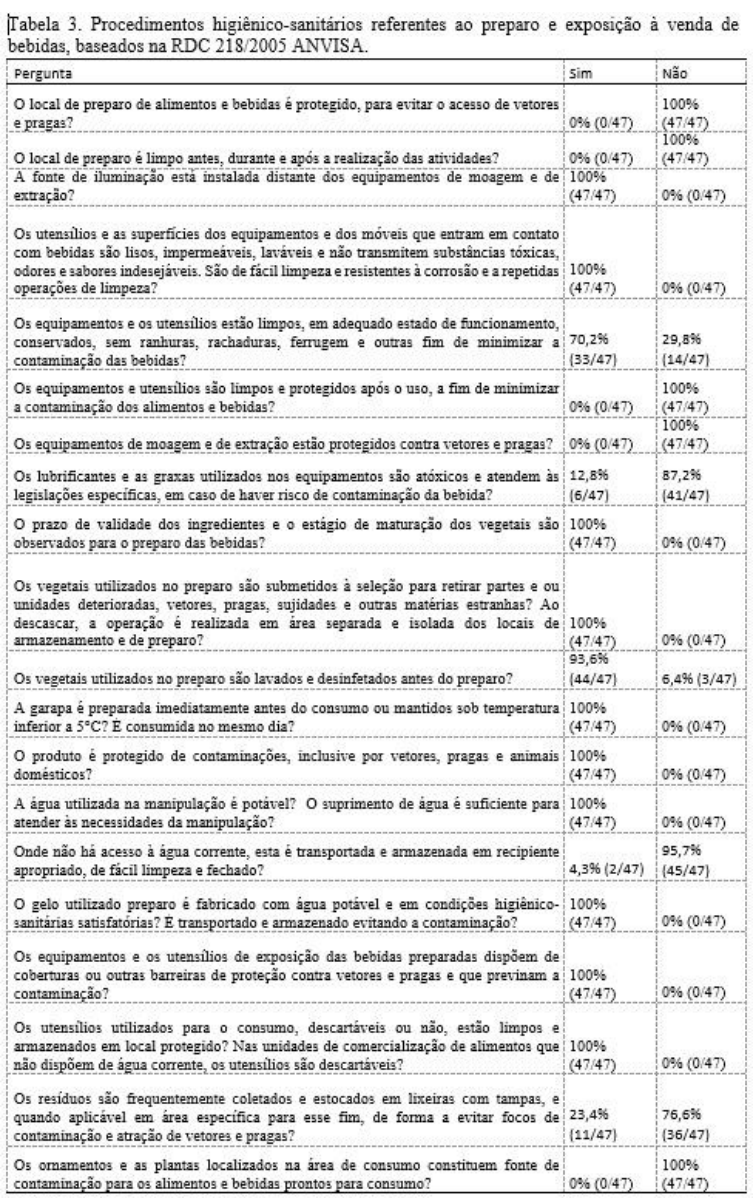

Em 93,6\% (44/47) dos casos, as cana de açúcar era lavadas e sanitizadas antes de serem processadas. A contaminação inicial da matéria-prima, influencia diretamente nos padrões higiênico-sanitários dos produtos vegetais processados (Medeiros et al., 2014). A higienização é essencial para a obtenção de um caldo de cana com elevada qualidade microbiológica. Em experimento realizado por Alves et al., (2008), toletes de cana sanitizados apresentaram redução de $99,9 \%$ na contagem de bolores, leveduras e enterobactérias. Compostos clorados orgânicos, como o dicloroisocianurato de sódio, possuem maior eficiência do que hipoclorito de sódio, pois não são inativados por matéria orgânica (Porto e Eiroa, 2006). Também são os mais indicados para o procedimento em alimentos vegetais, pois não deixam resíduos tóxicos a saúde do consumidor (Salomão et al., 2011).

Todos os comerciantes, 100\% (47/47), fazem a garapa para ser consumida no momento após sua produção, sendo que em 
casos onde isso não ocorre, a mesma é armazenada com gelo em jarras de plástico ou inox. Todavia, o problema é que o produto não atinge a temperatura de $5^{\circ} \mathrm{C}$ ou inferior prontamente, ocorrendo uma janela para a proliferação microbiana. $\mathrm{O}$ caldo de cana possui diversos nutrientes e condições propícias para o desenvolvimento de diversos patógenos (França, 2005). Estes microrganismos além de possibilitarem a ocorrência de DTAs, causam a deterioração do produto, modificando características organolépticas (Prado et al., 2010). A totalidade dos garapeiros, $100 \%$ (47/47), assegurou que o gelo elaborado com agua potável e em condições de acordo com as normas higiênico-sanitárias. Essa matériaprima é a principal fonte de contaminação com bactérias mesófilas aeróbicas, seja pela produção incorreta ou manipulação inadequada (Ferreira et al., 2014). Não se pode assegurar que os comerciantes utilizam gelo potável e os manuseiam corretamente, logo só deve-se consumir o ingrediente em locais seguros, onde se tenha segurança do alimento produzido (Peixoto e Pyrrho, 2010).

\section{CONCLUSÃO}

Os produtores de caldo de cana de Curitiba não estão obedecendo todos os itens constantes na RDC $n^{\circ} 218$, de 29 de julho de 2005, seja por desconhecimento do regulamento ou estarem agindo de má fé. Esta atitude expõem os consumidores à doenças transmissíveis por alimentos, tornando o produto um risco a saúde.

\section{NOTAS INFORMATIVAS}

Reservado ao parecer CEUA.

\section{REFERÊNCIAS}

ALMEIDA, M.D.; PENA, P.G.L. Feira livre e risco de contaminação alimentar: estudo de abordagem etnográfica em Santo Amaro,
Bahia. Revista Baiana de Saúde Pública, v.35, n.1, p.110-127, 2014.

ALVES, L.D.C.; ANDRADE, L.P.D.; GUIMARÃES, K.A.D.S. Treinamento sobre higiene e controle de qualidade para manipuladores de alimentos de uma Unidade de Alimentação e Nutrição. Higiene Alimentar, v.22, n.166/167, p.32-37, 2008.

ANDREOTTI, A.; BALERONI, F.H.; PAROSCHI, V.H.; PANZA, S.G.A. Importância do treinamento para manipuladores de alimentos em relação à higiene pessoal. Iniciação Científica Cesumar, v.5, n.1, p.29-33, 2007.

BRASIL. Ministério da Saúde. Agência Nacional de Vigilância Sanitária. Resolução - RDC n. ${ }^{\circ}$ 12, de 2 de janeiro de 2001. Regulamento técnico sobre padrões microbiológicos para alimentos. Diário Oficial [da] União, Brasília, 10 jan. 2001, Seção 1, p. 45-53. Disponível em: <http://www.anvisa.gov.br>. Acesso em: 20 out. 2014.

BRASIL. Ministério da Saúde. Agência Nacional de Vigilância Sanitária. Resolução - RDC n 218, de 29 de julho de 2005. Regulamento técnico de procedimentos higiênico-sanitários para manipulação de alimentos e bebidas preparados com vegetais. Diário Oficial [da] República Federativa do Brasil, Poder Executivo, Brasília, DF, 1 ago. 2005. Seção 1. Disponível em: <http://www.anvisa.gov.br>. Acesso em: 20 out. 2014.

CARDOSO, A.V.; LESCANO, S.A.; AMATO NETO, V.; GAKIYA, É.; SANTOS, S.V. Survival of Trypanosoma cruzi in sugar cane used to prepare juice. Revista do Instituto de Medicina Tropical de São Paulo, v.48, n.5, p.287-289, 2006.

DUARTE, D.L.; VIEIRA, D.A.D.P.; MACHADO, S.S.; VIANA, V.M. CALDO DE CANA CONSUMIDO EM VIAS PÚBLICAS-UMA REVISÃO. Cadernos de Educação, Tecnologia e Sociedade, v.2, n.1, p.1-4, 2011. 
FERREIRA, E. M.; LOPES, I. S.; PEREIRA, D. M.; RODRIGUES, L. C.; COSTA, F. N. Qualidade microbiológica do peixe serra (Scomberomerus brasiliensis) e do gelo utilizado na sua conservação. Arquivo do Instituto biológico, v.81, n.1, p.49-54, 2014.

FRANÇA, A.F. Avaliação da Qualidade Microbiológica do Caldo de Cana Comercializado na Área de Abrangência do Distrito Sanitário 5. Recife: UFRPE, 2005, 53p.

GANDRA, E. A.; REITEMBACH, A. F.; BOLANHO, B. C.; GUIMARÃES, J. S.; GANDRA, T. K.V. Condições Microbiológicas de Caldos de Cana Comercializados em Umuarama (PR). Revista Brasileira de Tecnologia Agroindustrial, v.1, n.2, p.61-69, 2007.

GENTA, T. M. S.; MAURÍCIO, A. A.; MATIOLI, G. Avaliação das Boas Práticas através de check-list aplicado em restaurantes self-service da região central de Maringá, Estado do Paraná. Acta Scientiarum Health Science, v.27, n.2, p.151-156, 2008.

IANNI, B.M.; MADY, C. The sugarcane juice was delicious, but... Arquivos Brasileiros de Cardiologia, v.85, n.6, p.379381, 2005.

JAY, J. M. Microbiologia moderna de los alimentos. Zaragoza : Acribia, 1992. 804 p.

LEAL, D. Crescimento da alimentação fora do domicílio. Segurança Alimentar $e$ Nutricional, v.17, n.1, p.123-132, 2010.

MEDEIROS, P.M.F.; SILVA, I.D.; MARQUES, F.P.P.; SOUZA, T.P.D. Qualidade físico-estrutural e aplicação de boas práticas de fabricação em lanchonetes localizadas na região central de AnápolisGO. Anuário da Produção de Iniciação Científica Discente, v.13, n.21, p.57-70, 2014.

NORBERG, A. N.; OLIVEIRA, J. T. M.; MONTEIRO, A. N. et al. Análise qualitativa e quantitativa de caldos de cana comercializados na região da Baixada
Fluminense, estado do Rio de Janeiro, Brasil, quanto à poluição por Staphylococcus aureus. Ciência \& Tecnologia, p.54-59, v.12, n. 2, 2012.

ODORIZZI, C.M.C.; GARCIA, L.; LEITE, C.C. Qualidade microbiológica de alimentos servidos em um hotel-resort do estado da Bahia. Higiene Alimentar, v.25, n.194/195, p.167-171, 2011.

OLIVEIRA, A.C.G.; NOGUEIRA, F.A.G.; ZANÃO, C.F.P.; SOUZA, C.W.O.; SPOTO, M.H.F. Análise das condições do comércio de caldo de cana em vias públicas de municípios paulistas. Segurança Alimentar e Nutricional, v.13, n.2, p.06-18, 2006.

PANZA, S.G.A.; BROTHERHOOD, R.; ANDREOTTI, A.; REZENDE, C.; BALERONI, F.H.; PAROSCHI, V.H. Avaliação das condições higiênico-sanitárias durante a manipulação dos alimentos, em um restaurante universitário, antes e depois do treinamento dos manipuladores. Higiene Alimentar, v.20, n.138, p.15-19, 2006.

PEIXOTO, S.T.; PYRRHO, A.D.S. Risco de transmissão de patógenos pelo uso de gelo. Higiene. Alimentar, v.26, n.206/207, p.98-102, 2012.

PEREIRA, L.; PINHEIRO, A.N.; SILVA, G.C. Manipulação Segura de alimentos. 2 ed. Rio de Janeiro: Senac Nacional, 2009, 86p.

PORTO, E.; EIROA, M.N.U. Avaliação da eficiência de sanificantes e do uso de atmosfera modificada sobre Listeria monocytogenes inoculada em alfaces. Brazilian Journal of Food Technology, v.9, n.3, p.177-183, 2006.

PRADO, S.D.P.T.; BERGAMINI, A.M.M., RIBEIRO, E.G.A., CASTRO, M.D.C.S.; OLIVEIRA, M.A.D. Avaliação do perfil microbiológico e microscópico do caldo de cana in natura comercializado por ambulantes. Revista do Instituto Adolfo Lutz, v.69, n.1, p.55-61, 2010.

SALOMÃO, B.D.C.M.; MÜLLER, C.; MASSAGUER, P.; ARAGÃ̃, G. Aplicação de dicloroisocianurato de sódio e ácido 
peracético para redução de esporos de Penicillium expansum, Byssochlamys fulva e Alicyclobacillus acidoterrestris na superfície de maçãs e em soluções aquosas. Alimentos e Nutrição Araraquara, v.22, n.2, p.219230,2011.

SANTOS, M.D.O.B.; RANGEL, V.P.; AZEREDO, D.P.; ALIMENTAR, S.; NUTRICIONAL, Q. Adequação de restaurantes comerciais às boas práticas. Higiene Alimentar, v.24, n.190/191, p.44-49, 2010.

SILVA, G. R. (2013). Percepção do conceito de higiene e segurança alimentar dos manipuladores de produtos cárneos de mercado público, Recife-PE, Brasil. Acta Veterinaria Brasilica, v.7, n.2, p.158-163.

SOUZA, L.H.L. A Manipulação Inadequada dos Alimentos: Fator de Contaminação. Higiene Alimentar, v.20, n.146, p.32-39, 2006.

STEINDEL, M.; DIAS, J.C.P.; ROMANHA, A.J. Doença de Chagas: mal que ainda preocupa. Ciência Hoje, v.37, p.32-38, 2005.

TORRES, S.A.M.; MIRANDA, A.D.S.; SILVA, V.A.; TOLEDO, S.C.; SILVA, M.A.; ROCHA, J.F. Treinamento de manipuladores de alimentos: merendeiras. Higiene Alimentar, v.20, n.143, p.33-36, 2006. 